Volume 19, No 3 International Journal of Radiation Research, July 2021

\title{
Histogram analysis of intravoxel incoherent motion and dynamic contrast-enhanced MRI with the two- compartment exchange model in glioma
}

\author{
F. Xing ${ }^{1}$ and G. Wu $\mathbf{u}^{2^{*}}$ \\ ${ }^{1}$ Department of Radiology, Renmin Hospital of Wuhan University, Wuhan 430060, P. R. China \\ ${ }_{2}^{2}$ Radiology Department, Shenzhen University General Hospital and Shenzhen University Clinical Medical Academy, \\ Shenzhen, 5180554, P. R. China
}

\section{- Original article}

\section{*Corresponding authors:}

Guangyao Wu, Ph.D.,

E-mail:

wuguangy2002@163.com

Revised: March 2020

Accepted: June 2020

Int. J. Radiat. Res., July 2021;

19(3): 505-514

DOI: $10.29252 /$ ijrr.19.2.505

\begin{abstract}
Background: Since different grades of gliomas have different treatment programs, prognosis, and survival rates, it's important to differentiate them effectively. Intravoxel incoherent motion (IVIM) and dynamic contrastenhanced (DCE) with the two-compartment exchange model (2CXM) have showed great potential for identifying the brain tumors. Materials and Methods: Thirty-nine patients with glioma underwent IVIM and DCE imaging at 3.0T. Quantitative parameters (mean, median, $10^{\text {th }}, 25^{\text {th }}, 75^{\text {th }}$ and $90^{\text {th }}$ percentiles) from IVIM (apparent diffusion coefficient (ADC), D, D*, f, and their product fD*) and DCE $\left(v_{p}, v_{e}, P S\right.$, and $\left.F_{p}\right)$ were analyzed. The independent Student's t-test and Mann-Whitney U-tests were used to assess whether these parameters could distinguish low- from high-grade glioma. Receiver-operating characteristic (ROC) curve analysis and Delong test were performed to determine and compare the diagnostic efficiency of IVIM and DCE parameters in differentiating low- and high-grade gliomas, respectively. Results: Various histogram statistics of IVIM and DCE parameters could differentiate different grades of glioma. $v_{e}$ and $v_{p}$ generally yielded higher area under the curve (AUC) values than IVIM parameters, and the differences in AUC values of $\mathrm{V}_{\mathrm{e}}$ mean and IVIM parameters had a statistical significance. Although DCE had higher AUC values than IVIM, they didn't reach statistical significance. Conclusions: $v_{e}$ was the best parameter in differential diagnosis of gliomas. IVIM had the similar diagnosis performance with DCE, and both IVIM and DCE-MRI parameters can be used for preoperative grading of gliomas.
\end{abstract}

Keywords: Apparent diffusion coefficient, Dynamic contrast-enhanced, Glioma, Intravoxel incoherent motion, $D$ value.

\section{INTRODUCTION}

Glioma is one of the most common primary tumors in the brain $(1,2)$, which arises from glial or precursor cells, and accounts for about $28 \%$ of all tumors and $80 \%$ of malignancies in the United States (3). Pathologically (4), glioma can be classified into four grades which are associated with different treatment programs, prognosis and survival rates. Compared to low-grade glioma, high-grade glioma usually requires further adjuvant chemoradiotherapy after surgery, and is relevant to poor prognosis and significantly decreased two-year survival rate. Therefore, a non-invasive imaging method for accurate preoperative grading of glioma would be conducive to select treatment program and determine prognosis.

Intravoxel incoherent motion (IVIM) imaging and dynamic contrast-enhanced (DCE-) MRI are two functional imaging techniques which are commonly employed in clinic for the assessment of tumor microenvironment. IVIM imaging was proposed by Le Bihan etal. (5) to study the 
microscopic translational motion of water molecules within two water compartments in the biological tissue: a slowly moving compartment in which the particles diffuse in a Brownian manner, and a rapidly moving compartment (a vascular compartment) where the water molecules move under the forced blood circulation (6). DCE-MRI employs the sequential T1-weighted imaging, and a contrast agent is injected to monitor the blood transport in the biological tissue, thus deriving the parameters pertaining to tissue perfusion $(7,8)$. Moreover, DCE-MRI with the two-compartment exchange model (2CXM) is more accurate. When compared with DCE-MRI, IVIM imaging has three conspicuous advantages:

(1) It can obtain diffusion and perfusion information simultaneously from biological tissues (9); (2) It is used widely and repeatedly in most patients without the need of contrast agent injection (10). (3) The post-processing of IVIM data is much simpler and faster. However, the clinical application of IVIM imaging is hampered by the sensitivity of IVIM algorithms to noise, and the incomplete interpretation of IVIM parameters in pathology (11).

The aims of this study are (1) to evaluate the differential diagnostic ability of IVIM and DCE-MRI with 2CXM model in different grades of gliomas; (2) to compare the parameters of these two methods and assess whether IVIM parameters are better than that of DCE.

\section{MATERIALS AND METHODS}

This retrospective study was approved by the local institutional review boards (IRBs) with a waiver for written informed consent. The registration number was 2017009 , and the date was July 2017.

\section{Patients}

A retrospective review of our institution database identified 168 patients with brain tumors who had undergone IVIM or DCE-MRI examination between November 2014 and February 2017. Among these patients, 39 patients were finally included in this study based on the following criterion: (1) patients with pathologically diagnosed glioma; (2) patients who had undergone IVIM and/or DCE-MRI examination prior to surgery or treatment; (3) the acquired images were complete and clear enough for post-processing.

Specifically, 38 patients had undergone IVIM, 35 patients had DCE-MRI, and 34 patients had both IVIM and DCE-MRI. Only some of 38 patients had gene tests for postoperative slices, so all 38 patients were pathology diagnosed according to the 2007 World Health Organization Tumor Classification (12). These patients (24 males, 15 females, the overall mean age was 46.9 years, range age $18-78$ years) were classified into: 1 case of grade I, 14 cases of grade II, 11 cases of grade III, and 13 cases of grade IV. The grade I and II gliomas were considered as low-grade gliomas (LGG) and the grade III and IV gliomas were high-grade gliomas (HGG).

\section{MR imaging and post-processing}

All images were acquired using a 3.0 Tesla (T) MR imaging unit (Trio; Siemens Medical Systems, Germany) with an eight-channel head array receiving coil for sensitivity encoding (SENSE) parallel imaging.

\section{Intravoxel incoherent motion MRI}

Diffusion-weighted (DW) imaging was performed using a two-dimensional (2D) single-shot spin-echo (SE) echo-planar sequence with the following parameters: repetition time/ echo time $(\mathrm{TR} / \mathrm{TE})=3000 / 88 \mathrm{~ms}, 90^{\circ}$ flip angle, number of excitations (NEX) $=1,13$ transverse sections, SENSE factor $=2$, slice thickness $/$ gap= $5 \mathrm{~mm} / 1 \mathrm{~mm}$, field of view (FOV) $=230 \times 230 \mathrm{~mm}$, $128 \times 128$ matrix, imaging time of $4 \mathrm{~min} 47 \mathrm{~s}$. Diffusion sensitizing gradients were all applied in the $x, y$, and $z$ directions with $55 \mathrm{~b}$-values $(0$, 50, 100, 150, 200, 300, 400, 500, 700, 900, 1000 $\mathrm{s} / \mathrm{mm}^{2}$ ). For comparison, several standard diagnostic MR images were also acquired, including three-dimensional (3D) T1-weighted, T2-weighted, fluid attenuation inversion recovery (FLAIR), and late contrast-enhanced 3D T1-weighted images.

Int. J. Radiat. Res., Vol. 19 No. 3, July 2021 


\section{Intravoxel incoherent motion data analysis}

The standard IVIM model is a double exponential model that accounts for diffusion and perfusion components in the tissue, which is expressed as equation 1 :

$\frac{\mathrm{S}_{\mathrm{b}}}{\mathrm{S}_{0}}=(1-\mathrm{f}) e^{-\mathrm{bD}}+\mathrm{f} e^{-\mathrm{b}\left(\mathrm{D}+\mathrm{D}^{*}\right)}$

As equation 1 shows, $S_{b}$ is the DW signal at non-zero $b$ value, $S_{0}$ is the signal strength $a t b=$ $0 \mathrm{~s} / \mathrm{mm}^{2}$. D is the tissue diffusion coefficient, $\mathrm{D}^{*}$ is a pseudo-diffusion coefficient associated with the blood movement in microvessels and $f$ is the perfusion fraction (13-15). A segmented (two-step) approach was employed for fitting the IVIM model. Firstly, the value of $\mathrm{D}$ was derived by fitting the high $b$ value $(\geq 200 \mathrm{sec} /$ $\mathrm{mm}^{2}$ ) data with a mono-exponential function, assuming negligible contribution of $\mathrm{D}^{*}$ in the high $b$ values $(2,16)$. Subsequently, with the D value fixed, $f$ and $D^{*}$ were estimated by non-linear regression fitting of all acquired $b$ values. In addition, the apparent diffusion coefficient (ADC) value was obtained by fitting a mono-exponential function using all b values.

\section{DCE-MRI}

DCE-MRI was performed by using a spoiled gradient recalled sequence with the following parameters: $\mathrm{TR} / \mathrm{TE}=3.32 / 0.92 \mathrm{~ms}, \mathrm{NEX}=1,10$ transverse sections, SENSE factor $=1.5$, slice thickness/gap $=5 \mathrm{~mm} / 1 \mathrm{~mm}, \mathrm{FOV}=240 \times 240$ $\mathrm{mm}, 224 \times 174$ matrix. 3 pre-contrast datasets were acquired using flip angles of $4^{\circ}, 8^{\circ}$ and $12^{\circ}$ (with 10 repetitions of each flip angle), followed by dynamic acquisition with a flip angle of $12^{\circ}$, which consisted of 150 measurements with temporal spacing of $2.0 \mathrm{~s}$. After the fifth dynamic acquisition, a gadolinium (Gd)-based contrast agent (Gadolinium-diethylene triamine pentaacetic acids (Gd-DTPA); Magnevist, Bayer HealthCare, Berlin, Germany) was injected through the antecubital vein at a rate of $4 \mathrm{~mL} / \mathrm{s}$ and a dose of $0.2 \mathrm{mmol} / \mathrm{kg}$ of body weight. Pre- and post- contrast T1-weighted imaging sequences were acquired in the same axial geometry. The total imaging time was $5 \mathrm{~min}$ $55 \mathrm{~s}$.

\section{DCE-MRI data analysis}

Voxel-level tissue concentration-time $C_{\text {tiss }}(\mathrm{t})$ curves were estimated from the DCE-MRI dataset using the variable flip angle method, and were fitted by using 2CXM, which could be described by the following equations $2-4$, and more specific principles were detailed in previous works $(7,17)$.

$$
\begin{aligned}
& C_{\text {tiss }}(t)=C_{A}(t) \otimes F_{p}[A \exp (\alpha t)+(1-A) \exp (\beta t)] \\
& \left(\begin{array}{l}
\alpha \\
\beta
\end{array}\right)=\frac{1}{2}\left[-\left(\frac{\mathrm{PS}}{\mathrm{V}_{\mathrm{p}}}+\frac{\mathrm{PS}}{\mathrm{V}_{\mathrm{e}}}+\frac{\mathrm{F}_{\mathrm{p}}}{\mathrm{V}_{\mathrm{p}}}\right) \pm \sqrt{\left.\left(\frac{\mathrm{PS}}{\mathrm{V}_{\mathrm{p}}}+\frac{\mathrm{PS}}{\mathrm{V}_{\mathrm{e}}}+\frac{\mathrm{F}_{\mathrm{p}}}{\mathrm{V}_{\mathrm{p}}}\right)^{2}-4 \frac{\mathrm{PS}}{\mathrm{V}_{\mathrm{e}}} \frac{\mathrm{F}_{\mathrm{p}}}{\mathrm{V}_{\mathrm{p}}}\right]}\right. \\
& A=\frac{\alpha+\frac{\mathrm{PS}}{\mathrm{V}_{\mathrm{p}}}+\frac{\mathrm{PS}}{\mathrm{V}_{\mathrm{e}}}}{\alpha-\beta}
\end{aligned}
$$

Where; $\otimes$ denotes convolution, $v_{e}$ denotes the fractional volume of extravascular extracellular space and $v_{p}$ is the fractional vascular volume. $F_{p}$ is the blood (plasma) flow in the intravascular compartment, and PS denotes a symmetric rate of contrast agent exchanging between both compartments. For each patient, the arterial input function (AIF) was manually selected with a feeding artery visible on one of the imaged slices. The voxel-level fitting of $C_{\text {tiss }}$ $(t)$ curves was performed and parametric maps of all kinetic parameters $\left(\mathrm{V}_{\mathrm{e}}, \mathrm{v}_{\mathrm{p}}, \mathrm{F}_{\mathrm{p}}\right.$, and PS) were generated according to Eq. 2-4.

\section{Regions of interest (ROIs)}

All image analyses were performed using MItalytics ${ }^{\circledR}$ software (FITPU Healthcare, Singapore; www.fitpuhealthcare.com). The tumor ROIs were manually delineated by an experienced neuroradiologist (G.Y.W. with more than 10 years of experience in neuroradiology) who was blinded to the pathologic results. Late contrast-enhanced 3D T1-weighted and T2-FLAIR images were referenced to identify the areas of solid tumor when drawing ROIs, and to avoid cystic, necrotic, hemorrhagic, or calcification areas and cerebrospinal fluid-filled regions. The ROIs were drawn on high $b$-value DW images (usually b=700 sec $/ \mathrm{mm}^{2}$ ) and on the 
mean DCE images (figure 1 and 2). For each patient, ROIs were manually drawn on three consecutive sections which contained the largest area of solid tumor, and the histogram analysis of each IVIM or DCE parameter was performed for the combined voxels within these ROIs.

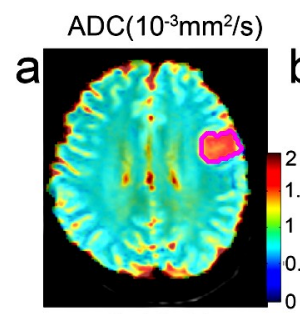

$v_{\mathrm{e}}(\mathrm{mL} / \mathrm{mL})$

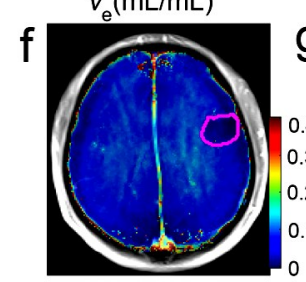

case (26-year-old man) with a grade II glioma in the left frontal lobe. Tumor ROI was shown in magenta on the ADC (a), IVIM (D (b), $\left.D^{*}(c), f(d), f D^{*}(e)\right)$ and DCE (ve (f), vp (g), Fp (h), and PS (i)) parameter maps, and on the mean DCE image computed from all contrast-enhanced images of the same slice (j).

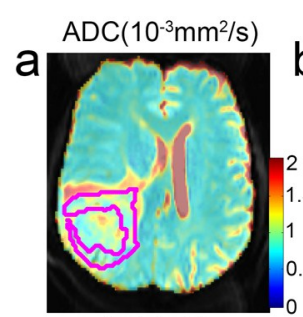

$v_{e}(\mathrm{~mL} / \mathrm{mL})$
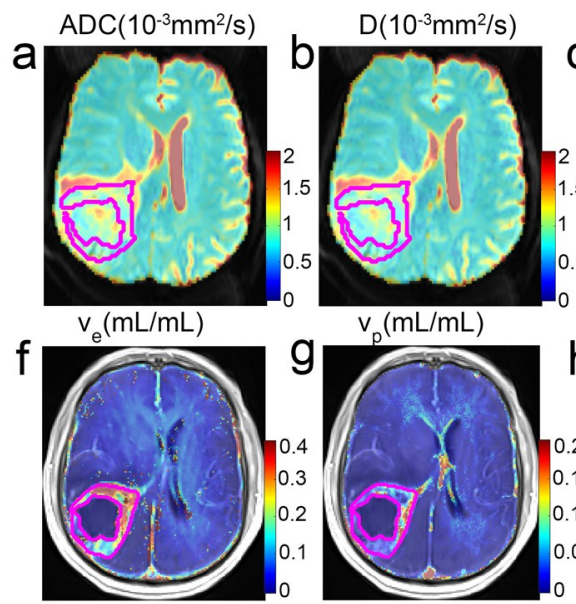

$v_{p}(\mathrm{~mL} / \mathrm{mL})$

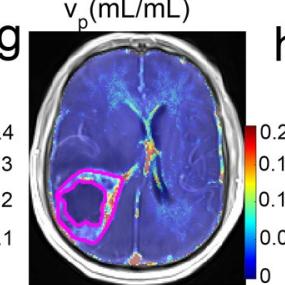

$\mathrm{D}^{*}\left(10^{-3} \mathrm{~mm}^{2} / \mathrm{s}\right)$

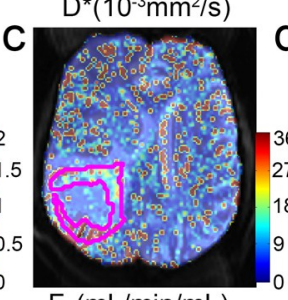

$\mathrm{F}(\mathrm{mL} / \mathrm{min} / \mathrm{mL})$
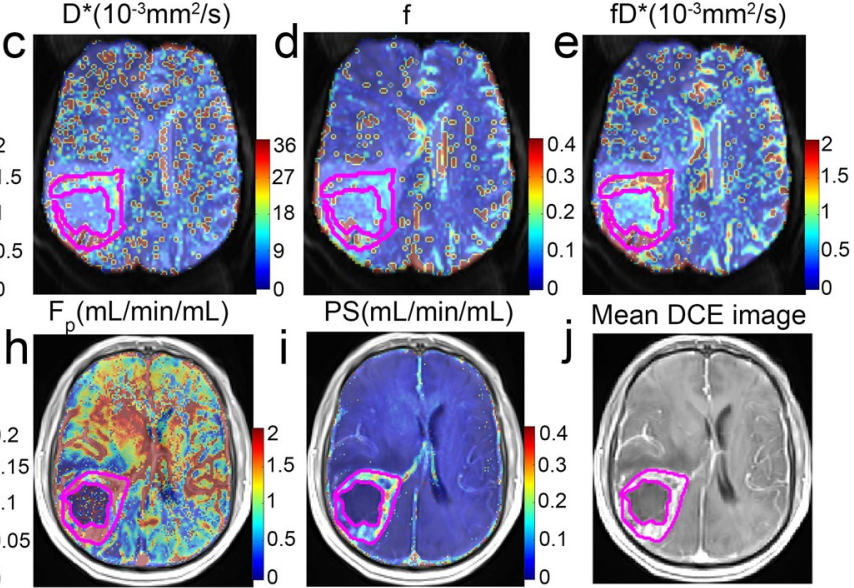

Figure 2. Example of a patient case (54-year-old man) with a grade IV glioma in the right pillow parietal lobe. Tumor ROI was shown in magenta on the ADC (a), IVIM (D (b), D* (c), f (d), fD*(e)) and DCE (ve (f), vp (g), Fp (h), and PS (i)) parameter maps, and on the mean DCE image computed from all contrast-enhanced images of the same slice (j).

\section{Statistical analyses}

For each patient, a particular IVIM or DCE parameter can be represented by the following voxel statistics which were derived from histogram analysis: (a) mean; (b) median; and (c) $10^{\text {th }}, 25^{\text {th }}, 75^{\text {th }}, 90^{\text {th }}$ percentiles. The two-tailed, independent Student's t-test was used for normally distributed variables, while Mann-Whitney U-tests were used for non-normally distributed variables. The receiver operating characteristic (ROC) curve analysis was performed to obtain the area under curve 508
(AUC), predicted cutoff value, sensitivity, and specificity of parameters that showed significant difference $(P<0.05)$ between low- and high-grade gliomas. To compare the ROC curves (i.e., diagnostic capacities) of IVIM- and DCE-derived parameters in differentiating between low- and high-grade gliomas, the method of Delong et al. (18) was used to test the statistical significance of the difference between AUC values of these parameters. We also explored whether multiple parameters of each functional imaging method could be combined to Int. J. Radiat. Res., Vol. 19 No. 3, July 2021 
yield ROC curves of higher AUC values than individual parameter. One-way analysis of variance (ANOVA) was applied to explore whether IVIM and DCE parameters could distinguish the grade II, III, IV gliomas. All statistical analyses were performed using SPSS (v. 19.0) and MedCalc (v. 15.2) software, and the statistical significance was defined at $P<0.05$.

\section{RESULTS}

\section{The value of IVIM parameters in differentiating low- and high-grade glioma}

As can be seen in table 1, high-grade gliomas exhibited significantly lower ADC values and D values, including the mean, median, $10^{\text {th }}, 25^{\text {th }}$, $75^{\text {th }}$, and $90^{\text {th }}$ percentile values, as compared

Table 1. Histogram analysis of IVIM parameters between LGG and HGG.

\begin{tabular}{|c|c|c|c|}
\hline Parameters & LGG(n=15) & HGG(n=23) & $P$ value \\
\hline \multicolumn{4}{|l|}{ Histogram ADC } \\
\hline Mean $\left(10^{-3} \mathrm{~mm}^{2} / \mathrm{s}\right)$ & $1.592 \pm 0.326$ & $1.218 \pm 0.324$ & $0.002^{a}$ \\
\hline Median $\left(10^{-3} \mathrm{~mm}^{2} / \mathrm{s}\right)$ & $1.589 \pm 0.358$ & $1.180 \pm 0.319$ & $0.001^{\mathrm{a}}$ \\
\hline $10^{\text {th }}\left(10^{-3} \mathrm{~mm}^{2} / \mathrm{s}\right)$ & $1.222 \pm 0.270$ & $0.941 \pm 0.244$ & 0.002 \\
\hline $25^{\mathrm{th}}\left(10^{-3} \mathrm{~mm}^{2} / \mathrm{s}\right)$ & $1.384 \pm 0.292$ & $1.041 \pm 0.266$ & 0.001 \\
\hline $75^{\text {th }}\left(10^{-3} \mathrm{~mm}^{2} / \mathrm{s}\right)$ & $1.788 \pm 0.426$ & $1.356 \pm 0.403$ & $0.003^{\mathrm{a}}$ \\
\hline $90^{\text {th }}\left(10^{-3} \mathrm{~mm}^{2} / \mathrm{s}\right)$ & $1.954 \pm 0.447$ & $1.551 \pm 0.487$ & $0.008^{a}$ \\
\hline \multicolumn{4}{|l|}{ Histogram D } \\
\hline Mean $\left(10^{-3} \mathrm{~mm}^{2} / \mathrm{s}\right)$ & $1.491 \pm 0.258$ & $1.159 \pm 0.310$ & $0.002^{\mathrm{a}}$ \\
\hline Median $\left(10^{-3} \mathrm{~mm}^{2} / \mathrm{s}\right)$ & $1.476 \pm 0.282$ & $1.121 \pm 0.305$ & $0.001^{\mathrm{a}}$ \\
\hline $10^{\text {th }}\left(10^{-3} \mathrm{~mm}^{2} / \mathrm{s}\right)$ & $1.139 \pm 0.238$ & $0.892 \pm 0.232$ & $0.002^{a}$ \\
\hline $25^{\mathrm{th}}\left(10^{-3} \mathrm{~mm}^{2} / \mathrm{s}\right)$ & $1.290 \pm 0.233$ & $0.989 \pm 0.252$ & 0.001 \\
\hline $75^{\text {th }}\left(10^{-3} \mathrm{~mm}^{2} / \mathrm{s}\right)$ & $1.670 \pm 0.334$ & $1.295 \pm 0.392$ & $0.003^{\mathrm{a}}$ \\
\hline $90^{\text {th }}\left(10^{-3} \mathrm{~mm}^{2} / \mathrm{s}\right)$ & $1.855 \pm 0.391$ & $1.476 \pm 0.461$ & $0.007^{\mathrm{a}}$ \\
\hline \multicolumn{4}{|l|}{ Histogram D* } \\
\hline Mean $\left(10^{-3} \mathrm{~mm}^{2} / \mathrm{s}\right)$ & $9.483 \pm 3.164$ & $13.500 \pm 5.766$ & 0.009 \\
\hline Median $\left(10^{-3} \mathrm{~mm}^{2} / \mathrm{s}\right)$ & $6.207 \pm 1.909$ & $7.840 \pm 1.973$ & 0.016 \\
\hline $10^{\text {th }}\left(10^{-3} \mathrm{~mm}^{2} / \mathrm{s}\right)$ & $2.603 \pm 2.651$ & $3.505 \pm 2.393$ & $0.075^{\mathrm{a}}$ \\
\hline $25^{\text {th }}\left(10^{-3} \mathrm{~mm}^{2} / \mathrm{s}\right)$ & $4.249 \pm 2.925$ & $5.531 \pm 1.579$ & $0.056^{\mathrm{a}}$ \\
\hline $75^{\text {th }}\left(10^{-3} \mathrm{~mm}^{2} / \mathrm{s}\right)$ & $9.393 \pm 3.36$ & $13.158 \pm 6.623$ & $0.062^{\mathrm{a}}$ \\
\hline $90^{\mathrm{th}}\left(10^{-3} \mathrm{~mm}^{2} / \mathrm{s}\right)$ & $16.780 \pm 7.403$ & $31.384 \pm 27.531$ & $0.184^{\mathrm{a}}$ \\
\hline \multicolumn{4}{|l|}{ Histogram f } \\
\hline Mean & $0.081 \pm 0.057$ & $0.093 \pm 0.033$ & $0.039^{\mathrm{a}}$ \\
\hline Median & $0.067 \pm 0.053$ & $0.080 \pm 0.021$ & $0.014^{\mathrm{a}}$ \\
\hline $10^{\text {th }}$ & $0.036 \pm 0.058$ & $0.025 \pm 0.018$ & $0.362^{a}$ \\
\hline $25^{\text {th }}$ & $0.057 \pm 0.069$ & $0.046 \pm 0.019$ & $0.324^{\mathrm{a}}$ \\
\hline $75^{\text {th }}$ & $0.113 \pm 0.075$ & $0.113 \pm 0.051$ & $0.411^{\mathrm{a}}$ \\
\hline $90^{\text {th }}$ & $0.151 \pm 0.095$ & $0.152 \pm 0.093$ & $0.709^{a}$ \\
\hline \multicolumn{4}{|l|}{ Histogram fD* } \\
\hline $\operatorname{Mean}\left(10^{-3} \mathrm{~mm}^{2} / \mathrm{s}\right)$ & $0.741 \pm 0.484$ & $0.983 \pm 0.487$ & 0.142 \\
\hline $\operatorname{Median}\left(10^{-3} \mathrm{~mm}^{2} / \mathrm{s}\right)$ & $0.515 \pm 0.403$ & $0.677 \pm 0.201$ & $0.014^{\mathrm{a}}$ \\
\hline $10^{\text {th }}\left(10^{-3} \mathrm{~mm}^{2} / \mathrm{s}\right)$ & $0.223 \pm 0.355$ & $0.163 \pm 0.136$ & $0.362^{\mathrm{a}}$ \\
\hline $25^{\mathrm{th}}\left(10^{-3} \mathrm{~mm}^{2} / \mathrm{s}\right)$ & $0.370 \pm 0.433$ & $0.346 \pm 0.157$ & $0.160^{\mathrm{a}}$ \\
\hline $75^{\text {th }}\left(10^{-3} \mathrm{~mm}^{2} / \mathrm{s}\right)$ & $0.931 \pm 0.573$ & $1.195 \pm 0.608$ & 0.189 \\
\hline $90^{\text {th }}\left(10^{-3} \mathrm{~mm}^{2} / \mathrm{s}\right)$ & $1.507 \pm 0.915$ & $2.036 \pm 1.348$ & 0.192 \\
\hline
\end{tabular}

Int. J. Radiat. Res., Vol. 19 No. 3, July 2021 with that of low-grade gliomas. To the contrary, low-grade gliomas exhibited much lower $\mathrm{D}^{*}$ and $\mathrm{f}$ (including the mean and median values) than that of high-grade gliomas, as well as the median value of $\mathrm{fD}^{*}$. All other parameters and histogram statistics exhibited no significant difference between these two groups (with $P>0.05$ ).

\section{The value of DCE parameters in differentiating low- and high-grade glioma}

As illustrated in table 2, as compared to high- grade gliomas, low-grade gliomas showed lower $v_{e}$ and $v_{p}$ values (including the mean, median, $10^{\text {th }}, 25^{\text {th }}, 75^{\text {th }}$, and $90^{\text {th }}$ percentiles), as well as PS value (mean, $10^{\text {th }}, 25^{\text {th }}$, and $75^{\text {th }}$ percentiles). However, all statistical representations of $F_{p}$ revealed no significant difference between the two groups.

Table 2. Histogram analysis of DCE parameters between LGG and HGG.

\begin{tabular}{|c|c|c|c|}
\hline Parameters & LGG & HGG & $P$ value \\
\hline \multicolumn{4}{|l|}{ Histogram $v_{p}$} \\
\hline Mean & $10.759 \pm 25.688$ & $22.905 \pm 21.02$ & 0.001 \\
\hline Median & $10.653 \pm 27.101$ & $21.715 \pm 23.605$ & 0.001 \\
\hline $10^{\text {th }}$ & $7.692 \pm 23.523$ & $9.794 \pm 10.467$ & 0.001 \\
\hline $25^{\text {th }}$ & $9.534 \pm 27.270$ & $15.096 \pm 15.729$ & 0.001 \\
\hline $75^{\text {th }}$ & $12.322 \pm 26.904$ & $29.760 \pm 27.259$ & 0.001 \\
\hline $90^{\text {th }}$ & $14.070 \pm 26.563$ & $37.254 \pm 31.581$ & 0.001 \\
\hline \multicolumn{4}{|l|}{ Histogram $v_{e}$} \\
\hline Mean & $5.529 \pm 5.417$ & $37.606 \pm 18.738$ & $<0.001$ \\
\hline Median & $4.055 \pm 4.664$ & $36.877 \pm 24.362$ & $<0.001$ \\
\hline $10^{\text {th }}$ & $1.315 \pm 1.430$ & $12.312 \pm 12.010$ & $<0.001$ \\
\hline $25^{\text {th }}$ & $2.376 \pm 2.444$ & $21.750 \pm 15.216$ & $<0.001$ \\
\hline $75^{\text {th }}$ & $6.282 \pm 7.668$ & $52.042 \pm 25.429$ & $<0.001$ \\
\hline $90^{\text {th }}$ & $11.387 \pm 12.06$ & $64.694 \pm 24.914$ & $<0.001$ \\
\hline \multicolumn{4}{|l|}{ Histogram PS } \\
\hline Mean & $22.768 \pm 21.913$ & $99.033 \pm 155.964$ & 0.009 \\
\hline Median & $11.664 \pm 14.592$ & $25.824 \pm 34.303$ & 0.076 \\
\hline $10^{\text {th }}$ & $2.750 \pm 3.687$ & $6.234 \pm 4.995$ & 0.007 \\
\hline $25^{\text {th }}$ & $5.783 \pm 7.278$ & $12.104 \pm 12.678$ & 0.048 \\
\hline $75^{\text {th }}$ & $22.042 \pm 26.354$ & $135.493 \pm 267.571$ & 0.034 \\
\hline $90^{\text {th }}$ & $64.188 \pm 81.618$ & $282.272 \pm 526.095$ & 0.060 \\
\hline \multicolumn{4}{|l|}{ Histogram $F_{p}$} \\
\hline Mean & $243.482 \pm 243.546$ & $318.086 \pm 293.809$ & 0.413 \\
\hline Median & $191.743 \pm 200.212$ & $268.037 \pm 267.713$ & 0.375 \\
\hline $10^{\text {th }}$ & $75.538 \pm 90.553$ & $126.600 \pm 118.071$ & 0.094 \\
\hline $25^{\text {th }}$ & $122.202 \pm 138.076$ & $182.549 \pm 170.034$ & 0.183 \\
\hline $75^{\text {th }}$ & $322.000 \pm 353.372$ & $391.013 \pm 387.559$ & 0.495 \\
\hline $90^{\text {th }}$ & $477.144 \pm 492.339$ & $583.735 \pm 554.562$ & 0.517 \\
\hline
\end{tabular}


ROC analysis of IVIM and DCE parameters for differentiating low- and high-grade glioma

As presented in table 3 and figure 3, the AUC value of combined parameters was higher than each parameter alone. The DCE parameters $v_{e}$ and $v_{p}$ generally achieved higher AUC values than diffusion parameters ADC and D. In particular, ADC_25 th and D_25 th showed the highest AUC values among the various statistical representations of diffusion parameters. Among the DCE parameters, $\mathrm{ve}_{-} 75^{\text {th }}$ had the highest AUC value with optimal threshold, sensitivity, and specificity of $25.813,86.4 \%$, and $100 \%$, respectively.

Besides, the $P$-values of Delong's test for comparing the AUC values of IVIM and DCE parameters were summarized in table 4 . The AUC value of $\mathrm{v}_{\mathrm{e}_{-}}$mean was higher than that of all
IVIM parameters, and had a significant difference (all $P<0.05$, except mean). It was observed that the AUC value of DCE method was higher than that of IVIM in table 3, but showing no significant difference.

\section{The value of IVIM and DCE parameters in} differentiating grade II 、 III and IV glioma

The ADC and D value (including the mean, median, $10^{\text {th }}, 25^{\text {th }}, 75^{\text {th }}$, and $90^{\text {th }}$ percentiles) exhibited significant difference between grade II and IV glioma, as well as the $\mathrm{D}^{*}$ _median. The $\mathrm{V}_{\mathrm{e}}$ value (including the mean, median, $10^{\text {th }}, 25^{\text {th }}$, $75^{\text {th }}$, and $90^{\text {th }}$ percentiles) could differentiate grade II from grade III and IV glioma, and all these results were displayed in figure 4 .

Table 3. ROC analysis of IVIM and DCE parameters in differentiating LGG from HGG.

\begin{tabular}{|c|c|c|c|c|c|c|c|c|c|}
\hline & AUC & $\begin{array}{c}\text { Cut-off } \\
\text { value }\end{array}$ & \begin{tabular}{|c|} 
Sensitivity \\
(\%)
\end{tabular} & \begin{tabular}{|c|}
$\begin{array}{c}\text { Specificity } \\
(\%)\end{array}$ \\
\end{tabular} & & AUC & $\begin{array}{c}\text { Cut-off } \\
\text { value }\end{array}$ & $\begin{array}{c}\text { Sensitivity } \\
(\%)\end{array}$ & \begin{tabular}{|c} 
Specificity \\
$(\%)$
\end{tabular} \\
\hline IVIM parameters & & & & & DCE Parameters & & & & \\
\hline $\begin{array}{c}\text { Histogram ADC } \\
\text { Mean }\left(10^{-3} \mathrm{~mm}^{2} / \mathrm{s}\right)\end{array}$ & 0.800 & 1.260 & 93.3 & 73.9 & Histogram $v_{p}$ & & & & \\
\hline Median $\left(10^{-3} \mathrm{~mm}^{2} / \mathrm{s}\right)$ & 0.812 & \begin{tabular}{|l|}
1.275 \\
\end{tabular} & 86.7 & 78.3 & Mean & 0.843 & 3.630 & 95.5 & 69.2 \\
\hline $10^{\text {th }}\left(10^{-3} \mathrm{~mm}^{2} / \mathrm{s}\right)$ & 0.803 & 1.045 & 73.3 & 82.6 & Median & 0.832 & 2.219 & 95.5 & 69.2 \\
\hline $25^{\text {th }}\left(10^{-3} \mathrm{~mm}^{2} / \mathrm{s}\right)$ & 0.826 & 1.104 & 93.3 & 69.6 & $10^{\text {th }}$ & 0.836 & 0.980 & 95.5 & 76.9 \\
\hline $75^{\text {th }}\left(10^{-3} \mathrm{~mm}^{2} / \mathrm{s}\right)$ & 0.791 & 1.330 & 93.3 & 69.6 & $25^{\text {th }}$ & 0.846 & 1.492 & 95.5 & 69.2 \\
\hline $90^{\text {th }}\left(10^{-3} \mathrm{~mm}^{2} / \mathrm{s}\right)$ & \begin{tabular}{|l|}
0.759 \\
0.875
\end{tabular} & \begin{tabular}{|c|}
1.473 \\
-
\end{tabular} & $\begin{array}{l}93.3 \\
95.3\end{array}$ & $\begin{array}{l}69.6 \\
69.2\end{array}$ & $75^{\text {th }}$ & 0.832 & 4.380 & 95.5 & 69.2 \\
\hline Histogram D & ( & & & & $90^{\text {th }}$ & 0.834 & 5.515 & 95.5 & 61.5 \\
\hline Mean $\left(10^{-3} \mathrm{~mm}^{2} / \mathrm{s}\right)$ & 0.797 & 1.220 & 93.3 & 73.9 & Combined $v_{p}$ & 0.916 & - & 85.7 & 84.6 \\
\hline Median $\left(10^{-3} \mathrm{~mm}^{2} / \mathrm{s}\right)$ & 0.813 & 1.155 & 93.3 & 73.9 & Histogram $v_{e}$ & & & & \\
\hline $10^{\text {th }}\left(10^{-3} \mathrm{~mm}^{2} / \mathrm{s}\right)$ & 0.797 & 0.997 & 80.0 & 82.6 & Mean & 0.955 & 17.630 & 86.4 & 100 \\
\hline$\frac{25^{\text {th }}\left(10^{-3} \mathrm{~mm}^{2} / \mathrm{s}\right)}{75^{\text {th }}\left(10^{-3} \mathrm{~mm}^{2} / \mathrm{s}\right)}$ & \begin{tabular}{|l|}
0.826 \\
0786 \\
\end{tabular} & \begin{tabular}{|l|}
1.043 \\
1267 \\
\end{tabular} & $\begin{array}{l}93.3 \\
93.3\end{array}$ & 73.9 & Median & 0.909 & 11.749 & 86.4 & 92.3 \\
\hline $90^{\text {th }}\left(10^{-3} \mathrm{~mm}^{2} / \mathrm{s}\right)$ & \begin{tabular}{|l|}
0.180 \\
0.762 \\
\end{tabular} & \begin{tabular}{|l|}
$1.20 /$ \\
1.417 \\
\end{tabular} & $\begin{array}{l}93.3 \\
93.3\end{array}$ & $\begin{array}{l}09.0 \\
73.9\end{array}$ & $10^{\text {th }}$ & 0.890 & 4.230 & 81.8 & 100 \\
\hline Combined D & 0.868 & - & 92.3 & 76.2 & $25^{\text {th }}$ & 0.916 & 7.430 & 86.4 & 100 \\
\hline Histogram D* & & & & & $75^{\text {th }}$ & 0.962 & 25.813 & 86.4 & 100 \\
\hline Mean $\left(10^{-3} \mathrm{~mm}^{2} / \mathrm{s}\right)$ & 0.717 & 10.95 & 69.6 & 73.3 & $90^{\text {th }}$ & 0.948 & 37.990 & 86.4 & 100 \\
\hline Median $\left(10^{-3} \mathrm{~mm}^{2} / \mathrm{s}\right)$ & 0.713 & 6.515 & 78.3 & 60.0 & Combined $v_{e}$ & 0.967 & - & 90.5 & 100 \\
\hline $\begin{array}{c}\text { Combined D* } \\
\text { Histogram f }\end{array}$ & 0.879 & - & 81.0 & 92.3 & Histogram PS & & & & \\
\hline Mean & 0.699 & 0.065 & 95.7 & 40.0 & Mean & 0.766 & 8.887 & 96.6 & 53.8 \\
\hline Median & 0.735 & 0.075 & 56.5 & 86.7 & $10^{\text {th }}$ & 0.774 & 2.072 & 86.4 & 76.9 \\
\hline Combined $\mathrm{f}$ & 0.842 & - & 90.5 & 76.9 & $25^{\text {th }}$ & 0.703 & 3.459 & 86.4 & 61.5 \\
\hline $\begin{array}{c}\text { Histogram fD* } \\
\text { Median }\left(10^{-3} \mathrm{~mm}^{2} / \mathrm{s}\right)\end{array}$ & & & & & $75^{\text {th }}$ & 0.717 & 10.277 & 90.9 & 61.5 \\
\hline$\frac{\left.\text { Median(10 } \mathrm{mm}^{2} / \mathrm{s}\right)}{\text { Combined } \mathrm{fD}^{*}}$ & \begin{tabular}{|l|}
0.738 \\
0.853 \\
\end{tabular} & \begin{tabular}{|c|}
0.495 \\
-
\end{tabular} & $\begin{array}{l}91.3 \\
95.3\end{array}$ & $\begin{array}{l}66.7 \\
76.9\end{array}$ & Combined PS & 0.967 & - & 97.3 & 84.6 \\
\hline IVIM & 0.971 & - & 99.8 & 92.3 & DCE & 0.998 & - & 99.8 & 100 \\
\hline
\end{tabular}

LGG: low-grade glioma; HGG: high-grade glioma; AUC: area under ROC curve; combined parameters meant the combination of the mean, median, $10^{\text {th }}, 25^{\text {th }}, 75^{\text {th }}$, and $90^{\text {th }}$ percentiles of each parameter. 

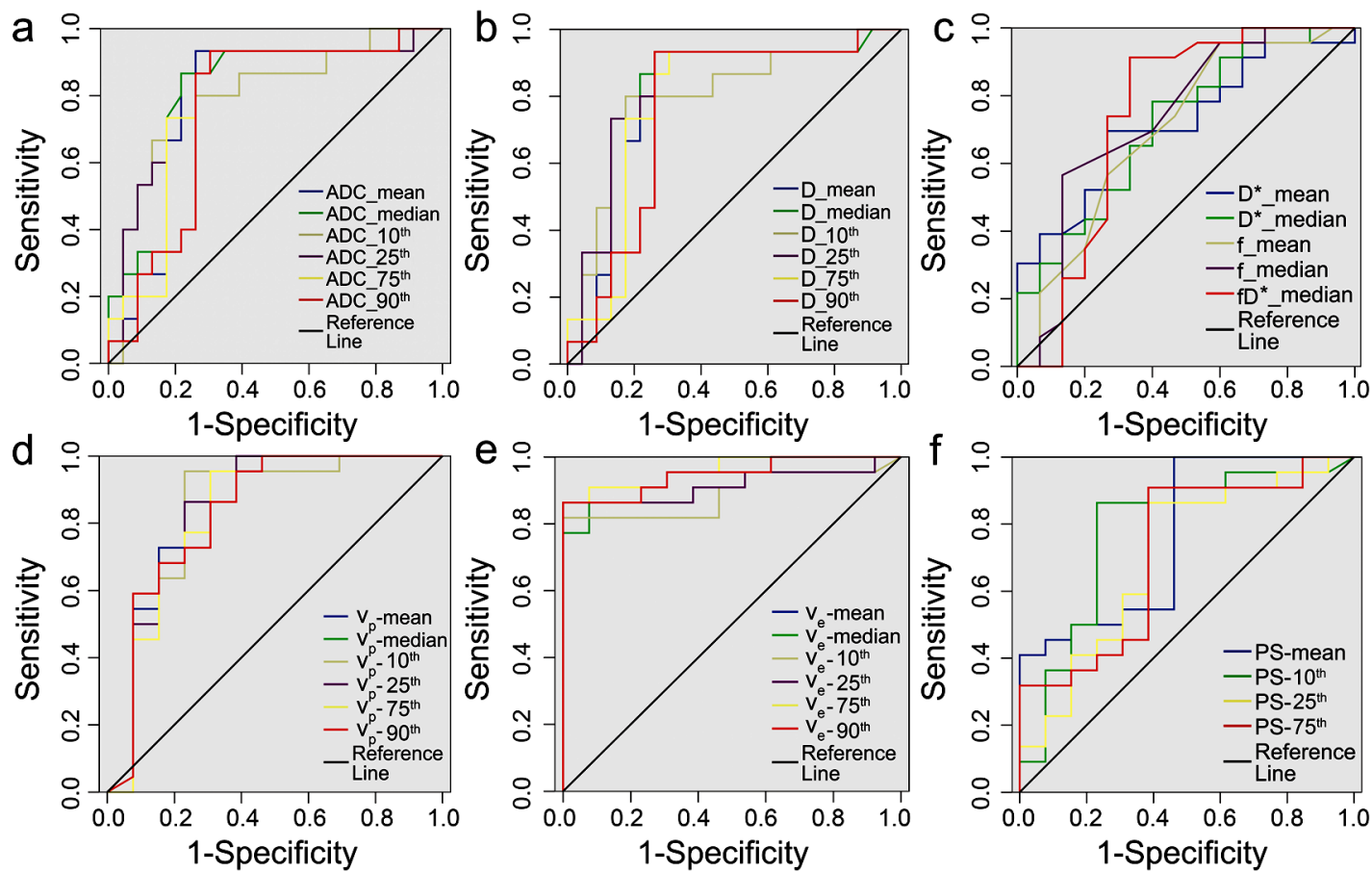

Figure 3. ROC curves of IVIM and DCE parameters in differentiating low-grade from high-grade glioma. All histogram statistics of $A D C(a)$ and $D(b)$ showed a significant difference between two groups; Some histogram statistics of $D^{*}$, $f$, and fD* (c) were higher in

high-grade than low-grade gliomas; High-grade gliomas had higher histogram statistics of vp (d), ve (e), and PS (f) than that of low-grade gliomas.

Table 4. The $P$ values of Delong test for comparing ROC curves of IVIM and DCE parameters in differentiating LGG from HGG.

\begin{tabular}{|c|c|c|c|c|c|c|c|c|c|c|}
\hline Parameters & \multicolumn{4}{|c|}{ Mean } & \multicolumn{5}{c|}{ Combine parameters } \\
\hline & $v_{p}$ & $v_{e}$ & $P S$ & $F_{p}$ & DCE & $v_{p}$ & $v_{e}$ & PS & $F_{p}$ & DCE \\
\hline ADC & 0.613 & 0.026 & 0.954 & 0.154 & - & 0.578 & 0.106 & 0.161 & 0.719 & - \\
\hline D & 0.594 & 0.024 & 0.978 & 0.173 & - & 0.492 & 0.061 & 0.132 & 0.801 & - \\
\hline$D^{*}$ & 0.356 & 0.007 & 0.75 & 0.217 & - & 0.575 & 0.146 & 0.214 & 0.728 & - \\
\hline$f$ & 0.550 & 0.050 & 0.978 & 0.167 & - & 0.471 & 0.134 & 0.136 & 1.000 & - \\
\hline$f^{*}$ & 0.236 & 0.009 & 0.736 & 0.291 & - & 0.458 & 0.176 & 0.241 & 0.903 & - \\
\hline IVIM & - & - & - & - & 0.074 & - & - & - & - & 0.336 \\
\hline
\end{tabular}

LGG: low-grade glioma; HGG: high-grade glioma; Combined parameters meant the combination of the mean, median, $10^{\text {th }}, 25^{\text {th }}, 75^{\text {th }}$, and $90^{\text {th }}$ percentiles of each parameter.

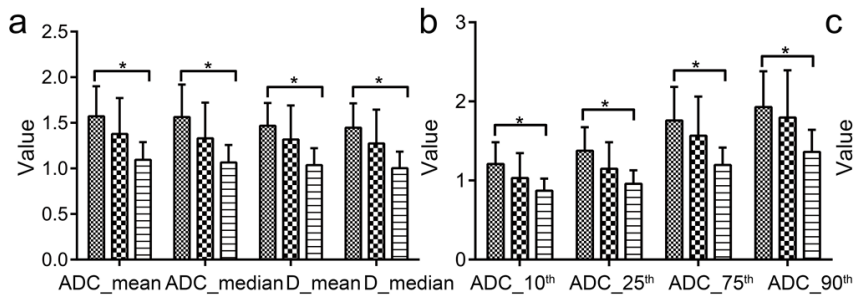

$\mathrm{d}$
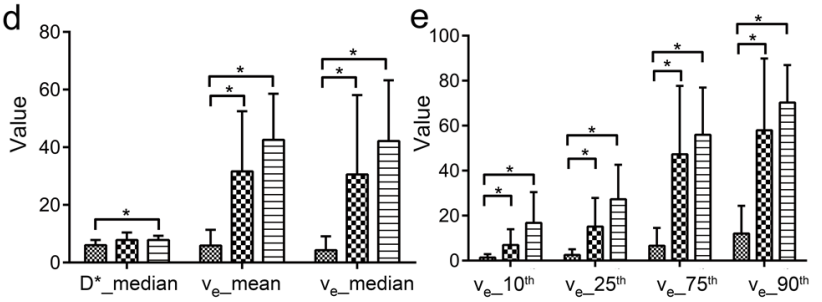

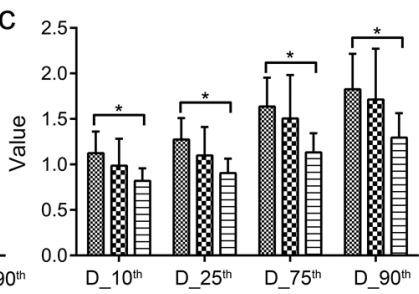

Grade II

回rade IV
Figure 4. Bar charts for comparison of IVIM and DCE parameters in discriminating grade II, III, and IV gliomas.

* indicates $\mathrm{P}<0.05$. The mean and median of ADC and $D$ value (a) could differentiate grade II from grade IV gliomas; The percentile values of $A D C$ (b) and D (c) showed a significant difference

between grade II and IV gliomas; The D*_median could differentiate grade II from grade IV gliomas, and ve_mean, and ve_median could differentiate grade II from grade III and IV glioma (d), as well as the percentile values of ve (e). 


\section{DISCUSSION}

Our data revealed that all histogram statistics of ADC and D values could be used to differentiate high- and low-grade gliomas, and ADC and D values were negatively correlated with glioma grade (grade IV < grade III < grade II) (table 1 and figure 4), which was consistent with the previous results (2). Water diffusion in biological tissues is affected by several factors such as cell size, cell density and cell geometry/ orientation (e.g., anisotropic diffusion of white matter) (15). High-grade gliomas typically exhibit increased tumor cell density, cell pleomorphism, nuclear fragmentation and microvascular proliferation. These factors could limit the diffusion of water molecules in tumor tissue, which in turn decrease the ADC and D value. According to Le Bihan (19), D could account for the water diffusion in the tissue more appropriately by excluding the effects of perfusion; while ADC encompassed both the effects of water diffusion and perfusion. In this study, the diagnostic efficiency of D was similar to $A D C$, which suggested that $\mathrm{D}$ may replace ADC in DWI to identify different grades of gliomas.

Previous studies have not established the effectiveness of $D^{*}$ in the differential diagnosis of glioma and cerebral tumors $(16,20)$, citing possible reasons of partial volume effects with the presence of CSF filling and/or necrotic regions. The results herein showed that the mean and median of $\mathrm{D}^{*}$ value were useful in differentiating between low- and high-grade gliomas, with the high-grade gliomas having higher $\mathrm{D}^{*}$ values (table 1 and figure 4 ). In this study, the ROIs of tumor were carefully delineated to exclude the cystic, necrotic, and cerebrospinal fluid-filled regions. Since $\mathrm{D}^{*}$ reflects the perfusion in the tumor, the higher $\mathrm{D}^{*}$ values exhibited by the high-grade glioma are consistent with the increase in microvascular blood flow in the more aggressive tumor which meets the growing demand for nutrients and oxygen. Both $\mathrm{D}^{*}$ and $\mathrm{v}_{\mathrm{p}}$ were found to increase in high-grade gliomas when compared with low-grade gliomas. Meanwhile, a moderate positive correlation existed between $\mathrm{D}^{*}$ _mean and $\mathrm{v}_{\mathrm{p} \_}$mean in gliomas (table 4 ).
IVIM imaging proposes to separate perfusion from tissue water diffusion in the DWI signal, with the perfusion fraction $f$ denoting the relative proportion of signal given by the vascular component. $f$ is clinically useful to diagnose cerebral diseases, especially brain tumors. For example, Shen et al. (2) showed that $\mathrm{f}$ could distinguish between low-grade and high-grade gliomas, as well as grade II, III, IV gliomas (mean \pm standard deviation, $0.076 \pm$ 0.016 versus $0.105 \pm 0.023$ versus $0.113 \pm 0.019$, $\mathrm{P}<0.001$ ). Shim et al. (21) also found that $\mathrm{f}$ values between metastasis, glioblastoma, and primary central nervous system lymphoma were statistically significant $(P<0.05)$. Suh et al. (22) revealed that $f$ values of glioblastoma and atypical primary central nervous system lymphoma were significantly different (reader 1 , $0.101 \pm 0.016$ [standard deviation] vs $0.021 \pm$ $0.010 ; P \neg 4.445$; reader $6: 4.54^{1} / 4.468$ vs $4.46^{1}$ \pm 0.015; $P<0.001$ ). All these results were similar to our observation that a significant difference in $\mathrm{f}$ value existed between high-grade and low-grade glioma. The increased neovascularization would result in high perfusion, which supported that high-grade gliomas tend to have increased $\mathrm{f}$ and $\mathrm{fD}^{*}$ values as compared to low-grade gliomas.

$v_{e}$ and $v_{p}$ refer to the fractional interstitial and vascular volumes of the tumor, respectively, which are assessed by DCE. From table 2, it could be seen that all histogram statistics of $\mathrm{v}_{\mathrm{e}}$ and $v_{p}$ in high-grade glioma were significantly higher than that of low-grade gliomas. This agreed well with previous studies that $\mathrm{v}_{\mathrm{e}}$ could discriminate low- from high- grade glioma (23-25). An increase in $v_{e}$ in high-grade gliomas could be attributed to the possible development of necrotic regions as the tumor progressed. However, previous studies did not reveal the significance of $\mathrm{v}_{\mathrm{p}}$ in differentiating gliomas. A higher $v_{p}$ value for high-grade gliomas in current work was concordant with the observation that microvascular proliferation in high-grade gliomas was more prominent than low-grade gliomas, with the high-grade gliomas having higher vascular density. The AUC values of $v_{e}$ and $v_{p}$ were generally higher than IVIM parameters (table 3 and figure 3), and the

Int. J. Radiat. Res., Vol. 19 No. 3, July 2021 
improvement in AUC value of ve_mean had statistical significance (table 4), which indicated that $v_{e}$ would be the best parameter in differential diagnosis of low- and high-grade gliomas.

This study reported that the AUC value of combined parameters was higher than that of individual parameter, as well as the AUC value that combined all parameters with each functional imaging method and each parameter. Thus, it implied that to use the AUC value with combined parameters to distinguish different grades of gliomas may be better. Although the AUC value of DCE was slight higher than that of IVIM, the improvement in AUC value did not reach statistical significance for current dataset, indicating similar diagnostic efficiency for IVIM and DCE methods. From a practical implementation perspective, IVIM may be preferred over DCE clinically in the differential diagnosis of glioma, because IVIM imaging does not require the injection of gadolinium contrast. Moreover, the processing of IVIM data is much simpler than DCE-MRI which doesn't require to select an appropriate AIF.

However, our research also had some limitations. Firstly, the number of patients in this study was relatively small, which could potentially give rise to wider/overlapping confidence intervals in the analysis of various grades of gliomas. Secondly, due to machine limitations, only four low b-values $(<200 \mathrm{sec} /$ $\mathrm{mm}^{2}$ ) were acquired during IVIM imaging. The acquisition of additional low b-values should improve the fitting and stability of IVIM perfusion parameters $\left(D^{*}, f\right.$, and $\left.\mathrm{fD}^{*}\right)$, which could possibly strengthen the statistical significance. Thirdly, the IVIM and DCE images were not available for all cases in this study, which would otherwise bring about a more direct comparison of these two methods.

In conclusion, our study showed that various histogram statistics of IVIM and DCE parameters could distinguish between low- and high-grade gliomas. The combined parameters had higher AUC value than that of individual parameter, as well as the AUC value that combined all parameters with each functional imaging method and each parameter. $v_{e}$ and $v_{p}$ had higher diagnosis ability than IVIM parameters, and $v_{e}$ was the best parameter in differential diagnosis of gliomas. IVIM had the similar diagnosis performance with DCE, and both of them could potentially be used for preoperative grading of gliomas.

\section{ACKNOWLEDGEMENTS}

This study was funded by the National Natural Science Foundation of China (Grant No. 2016YFC1304702), the Fundamental Research Funds for the Central Universities (2042020kf0126) and Shenzhen University Presidential Fund (85706-0000040544)"

Conflicts of interest: Declared none.

\section{REFERENCES}

1. Lin $Y$, Li J, Zhang Z, Xu Q, Zhou Z, Zhang Z, Zhang Y, Zhang Z (2015) Comparison of intravoxel incoherent motion diffusion-weighted MR imaging and arterial spin labeling MR imaging in gliomas. BioMed Research International, 2015: 234245.

2. Shen $N$, Zhao L, Jiang J, Jiang $R$, Su C, Zhang S, Tang $X$, Zhu $W$ (2016) Intravoxel incoherent motion diffusion-weighted imaging analysis of diffusion and microperfusion in grading gliomas and comparison with arterial spin labeling for evaluation of tumor perfusion. J Magn Reson Imaging, 44: 620-632.

3. Ostrom QT, Gittleman $\mathrm{H}$, Liao P, Rouse $\mathrm{C}$, Chen $\mathrm{Y}$, Dowling J, Wolinsky Y, Kruchko C, Barnholtz-Sloan J (2014) CBTRUS statistical report: primary brain and central nervous system tumors diagnosed in the United States in 2007-2011. Neuro-Oncology, (16)4: iv1-63.

4. Louis DN, Perry A, Reifenberger G, von Deimling A, Figarella-Branger D, Cavenee WK, Ohgaki H, Wiestler OD, Kleihues P, Ellison DW (2016) The 2016 world health organization classification of tumors of the central nervous system: a summary. Acta Neuropathologica, 131: 803-820.

5. Le Bihan D (1988) Intravoxel incoherent motion imaging using steady-state free precession. Magnetic Resonance in Medicine, 7: 346-351.

6. Puig J, Sanchez-Gonzalez J, Blasco G, Daunis IEP, Federau C, Alberich-Bayarri A, Biarnes C, Nael K, Essig M, Jain R, Wintermark M, Pedraza S (2016) Intravoxel incoherent motion metrics as potential biomarkers for survival in glioblastoma. PloS One, 11: e0158887. 
7. Koh TS, Bisdas S, Koh DM, Thng CH (2011) Fundamentals of tracer kinetics for dynamic contrast-enhanced MRI. J Magn Reson Imaging, 34: 1262-1276.

8. Jackson A, O'Connor JP, Parker GJ, Jayson GC (2007) Imaging tumor vascular heterogeneity and angiogenesis using dynamic contrast-enhanced magnetic resonance imaging. Clin Cancer Res, 13: 3449-3459.

9. Kim HS, Suh CH, Kim N, Choi CG, Kim SJ (2014) Histogram analysis of intravoxel incoherent motion for differentiating recurrent tumor from treatment effect in patients with glioblastoma: initial clinical experience. AJNR Am J Neuroradiol, 35: 490-497.

10. Federau C, Cerny M, Roux M, Mosimann PJ, Maeder P, Meuli R, Wintermark M (2017) IVIM perfusion fraction is prognostic for survival in brain glioma. Clinical Neuroradiology, 27: 485-492.

11. Stieb S, Boss A, Wurnig MC, Ozbay PS, Weiss T, Guckenberger M, Riesterer O, Rossi C (2016) Non-parametric intravoxel incoherent motion analysis in patients with intracranial lesions: Test-retest reliability and correlation with arterial spin labeling. Neurolmage Clinical, 11: 780788.

12. Louis DN, Ohgaki H, Wiestler OD, Cavenee WK, Burger PC, Jouvet A, Scheithauer BW, Kleihues P (2007) The 2007 WHO classification of tumours of the central nervous system. Acta Neuropathologica, 114: 97-109.

13. Wirestam R, Borg M, Brockstedt S, Lindgren A, Holtas $S$, Stahlberg $F$ (2001) Perfusion-related parameters in intravoxel incoherent motion MR imaging compared with CBV and CBF measured by dynamic susceptibility-contrast MR technique. Acta Radiologica, 42: 123-128.

14. Chandarana H, Kang SK, Wong S, Rusinek H, Zhang JL, Arizono S, Huang WC, Melamed J, Babb JS, Suan EF, Lee VS, Sigmund EE (2012) Diffusion-weighted intravoxel incoherent motion imaging of renal tumors with histopathologic correlation. Investigative Radiology, 47: 688-696.

15. lima M and Le Bihan D (2016) Clinical intravoxel incoherent motion and diffusion MR imaging: past, present, and future. Radiology, 278: 13-32.

16. Kim DY, Kim HS, Goh MJ, Choi CG, Kim SJ (2014) Utility of intravoxel incoherent motion MR imaging for distinguishing recurrent metastatic tumor from treatment effect following gamma knife radiosurgery: initial experience. AJNR Am J Neuroradiol, 35: 2082-2090.

17. Khalifa F, Soliman A, El-Baz A, Abou El-Ghar M, El-Diasty T, Gimel'farb G, Ouseph R, Dwyer AC (2014) Models and methods for analyzing DCE-MRI: a review. Medical Physics, 41: 124301.

18. DeLong ER, DeLong DM, Clarke-Pearson DL (1988) Comparing the areas under two or more correlated receiver operating characteristic curves: a nonparametric approach. Biometrics, 44: 837-845.

19. Le Bihan D, Breton E, Lallemand D, Aubin ML, Vignaud J, Laval-Jeantet M (1988) Separation of diffusion and perfusion in intravoxel incoherent motion MR imaging. Radiology, 168: 497-505.

20. Togao O, Hiwatashi A, Yamashita K, Kikuchi K, Mizoguchi M, Yoshimoto K, Suzuki SO, Iwaki T, Obara M, Van Cauteren $\mathrm{M}$, Honda $\mathrm{H}$ (2016) Differentiation of high-grade and low-grade diffuse gliomas by intravoxel incoherent motion MR imaging. Neuro-Oncology, 18: 132-141.

21. Shim WH, Kim HS, Choi CG, Kim SJ (2015) Comparison of apparent diffusion coefficient and intravoxel incoherent motion for differentiating among glioblastoma, metastasis, and lymphoma focusing on diffusion-related parameter. Plos One, 10: e0134761.

22. Suh CH, Kim HS, Lee SS, Kim N, Yoon HM, Choi CG, Kim SJ (2014) Atypical imaging features of primary central nervous system lymphoma that mimics glioblastoma: utility of intravoxel incoherent motion MR imaging. Radiology, 272: 504-513.

23. Choi HS, Kim AH, Ahn SS, Shin NY, Kim J, Lee SK (2013) Glioma grading capability: comparisons among parameters from dynamic contrast-enhanced MRI and ADC value on DWI. Korean Journal of Radiology, 14: 487-492.

24. Li X, Zhu Y, Kang H, Zhang $Y$, Liang $H$, Wang S, Zhang W (2015) Glioma grading by microvascular permeability parameters derived from dynamic contrast-enhanced MRI and intratumoral susceptibility signal on susceptibility weighted imaging. Cancer Imaging, 15: 4.

25. Abe T, Mizobuchi $Y$, Nakajima K, Otomi $Y$, Irahara $S$, Obama $Y$, Majigsuren M, Khashbat D, Kageji T, Nagahiro $S$, Harada M (2015) Diagnosis of brain tumors using dynamic contrast-enhanced perfusion imaging with a short acquisition time. SpringerPlus, 4: 88. 